\title{
Unravelling the Protective Effects of Emodin Against Cyclophosphamide Induced Gonadotoxicity in Male Wistar Rats
}

\author{
Yinhua Wang' \\ Zhaoling Zou' \\ Amit Jaisi $\mathbb{D}^{2}$ \\ Opeyemi Joshua Olatunji (iD
}

'The Second Peoples Hospital of Wuhu, Wuhu, 24I00I, Anhui, People's Republic of China; ${ }^{2} \mathrm{School}$ of Pharmacy, Walailak University, Nakhon Si Thammarat, 80I60, Thailand; ${ }^{3}$ Faculty of Thai Traditional Medicine, Prince of Songkla University, Hat Yai, 901 10, Thailand
Correspondence: Opeyemi Joshua Olatunji

Faculty of Thai Traditional Medicine, Prince of Songkla University, Hat Yai, 90I 10, Thailand

Tel +6674282722

Email opeyemi.j@psu.ac.th
Background: Over the past few decades, cyclophosphamide (CP) has been extensively used as a broad-spectrum alkylating agent for the treatment of various cancers and solid tumors. However, the therapeutic actions on $\mathrm{CP}$ are not limited to only cancer cells, as it simultaneously exerts significant toxicities on healthy cells through the instigation of oxidative stress and oxidative damages. CP induced testicular toxicity is associated with impaired spermatogenesis, reduced sperm functionality, reproductive hormone and testicular weight. This study was aimed at unravelling the protective effects of emodin (EMD) on testicular toxicity following CP treatment.

Methods: Twenty-four male Wistar rats were allotted into 4 groups as normal control group (NCG), CP control group (CPCG), EMD25+CP (25 mg/kg in 5\% tween 80) and EMD50+CP groups $(50 \mathrm{mg} / \mathrm{kg}$ in $5 \%$ tween 80$)$. EMD was orally administered for 35 consecutive days, while four doses of CP (100 mg/kg/week) were administered intraperitoneally from the second to fifth week of treatment. Thereafter, the animals were sacrificed and histopathological examination of the testes as well as serum/testicular biochemical assays were conducted.

Results: The results revealed that $\mathrm{CP}$ significantly impeded sperm function parameters including sperm count, viability and motility as well as decreased reproductive hormones (testosterone, $\mathrm{LH}$ and FSH) levels. In addition, $\mathrm{CP}$ enhanced testicular oxidative stress and proinflammatory markers (MDA, IL-6 and TNF- $\alpha$ ), while simultaneously decreasing testicular antioxidant enzymes (GSH, GPx, SOD and CAT). Evidence of marked histopathological alterations was also observed in the H\&E stained testicular tissues of $\mathrm{CP}$ treated rats. EMD significantly prevented these CP induced negative effects.

Conclusion: This study provides a basis for the potential use of EMD in counteracting chemotherapy induced testicular toxicity. The results further suggest that EMD testicular protective effects in CP-treated rats may be mediated through its modulatory role on oxidative stress and inflammation.

Keywords: emodin, cyclophosphamide, testicular toxicity, oxidative stress, inflammation

\section{Introduction}

Cancer is one of the most prevalent disease that affects a huge population globally and it notably impairs the quality of life as well as the life expectancy of cancer patients. ${ }^{1}$ In fact, according to the World Health Organization (WHO), cancer is the leading cause of mortality before the age of 70 in over 100 countries. ${ }^{2}$ However, in the past few decades, significant strides have been achieved in the development of potent anticancer agents including platinum-based anticancer drugs like cisplatin, 
carboplatin and oxaliplatin as well as nitrogen mustard antitumor agents such as chlorambucil and cyclophosphamide. ${ }^{1,3,4}$ These anticancer drugs have significantly improved the life expectancy of cancer patients. Unfortunately, most of these agents indiscriminately target both cancer and normal cells, thus posing severe and in extreme cases life-threatening toxicities. ${ }^{5-7}$

Cyclophosphamide (CP) is a potent cytotoxic alkylating agent that is widely used in chemotherapy for treating various cancers including breast, ovarian, small cell lung, brain and blood cancers. CP also induces significant toxicities including severe bladder bleeding, nephrotoxicity, gonadotoxicity and cardiotoxicity, which has significantly constrained its clinical usefulness. ${ }^{8,9}$ CP predisposes female patients to premature menopause, while the risk of irreversible infertility in both male and female patients is significantly increased upon exposure to cumulative drug dose. ${ }^{10}$ The testes is a major reproductive organ and it mainly responsible for the production of androgens such as testosterone and spermatozoa needed for reproductive functions. ${ }^{11} \mathrm{CP}$ induced testicular toxicity is associated with impaired spermatogenesis, reduced sperm functionality, reproductive hormone and testicular weight. $^{12,13}$ Several studies have suggested that acrolein, an enzymatic toxic metabolite of $\mathrm{CP}$ promotes the generation of reactive oxygen species and lipid peroxidation, thus inducing oxidative stress and oxidative damage. ${ }^{12}$ Furthermore, the abundance of polyunsaturated fatty acids as well as the low levels of antioxidants in the testes and spermatozoa exposes the tissues to oxidative stress and damages. ${ }^{12,13}$ Unfortunately, despite the widely reported testicular toxicity associated with $\mathrm{CP}$, there is no approved agent till date to counteract $\mathrm{CP}$-induced gonadotoxicity.

Natural product-based medicinal agents have gained significant attention in recent decades as potent and relatively safer alternatives for the treatment of various diseases and as pharmacological agents that can counteract drug-induced toxicities. ${ }^{14}$ A lot of these phytochemicals have displayed broad-spectrum activities particularly antiinflammatory and antioxidant properties, which have basically set a foundation for their therapeutic efficacy against disorders that have ROS and oxidative stress has their main pathophysiology. ${ }^{15,16}$ Therefore, their potential as preventive agents against chemotherapy-induced toxicities cannot be over emphasised. ${ }^{17}$ Emodin (EMD) is a bioactive naturally occurring anthraquinone found in several plant and fungal species, and it has been reported to have several biological properties including antineoplastic, antioxidant, anti-inflammatory, antidiabetic, hepatoprotective and anti-angiogenic properties. ${ }^{18}$ Previous studies have reported the protective properties of emodin against cisplatin-induced nephrotoxicity, while another study suggested that emodin showed hepatoprotective effects against $\mathrm{CCl}_{4}$-induced hepatic injury in rats via antioxidant effects. ${ }^{19-21}$ Furthermore, recently Zhao et al demonstrated that emodin attenuated CP induced oxidative damage in peripheral blood leukocytes of blunt snout bream through its ability to upregulate antioxidant prowess. $^{22}$ However, there are no reports suggesting the protective effects of emodin on $\mathrm{CP}$-induced organ toxicities. Keeping this in mind, this study explored the protective potency of emodin against cyclophosphamide-induced gonadotoxicity.

\section{Materials and Methods}

\section{Animals}

Seven weeks old twenty four male Wistar rats were used for the experiment. The animals were specific pathogen free and kept in separate cages with six rats per cage. The environmental conditions including temperature, relative humidity as well as the light/dark period were in accordance with standard procedures. ${ }^{5}$ The rats were acclimatized for a week and were afforded standard rat food and water ad libitum. The procedures used in this experiment were approved by the Animal Ethics Committee of The Second Peoples Hospital of Wuhu (Ethic code: Wuhuey20201008). Additionally, the guidelines of the National Institutes of Health guide for the care and use of laboratory animals (NIH Publications No. 8023, revised 1978) were strictly followed.

\section{Experimental Protocol/Treatment Protocol}

All the animals were grouped for treatment as follows: normal control group (NCG), CP control group (CPCG), low dose emodin group (CP+EMD25) and high dose emodin group (CP+EMD50). The rats in $\mathrm{NCG}$ and $\mathrm{CPCG}$ groups were given $5 \%$ tween 80 on a daily basis for 5 weeks. At the same time, the rats in EMD25+CP and EMD50+CP groups were administrated with daily doses of 25 and $50 \mathrm{mg} / \mathrm{kg}$ of emodin, respectively, for 5 weeks. From the second week of treatment, all the rats except for the NCG group were intraperitoneally injected with CP $(100 \mathrm{mg} / \mathrm{kg})$ once a week and continued for 4 weeks. ${ }^{23}$ An overview of the experimental design is shown in Figure 1. The concentration of CP and 


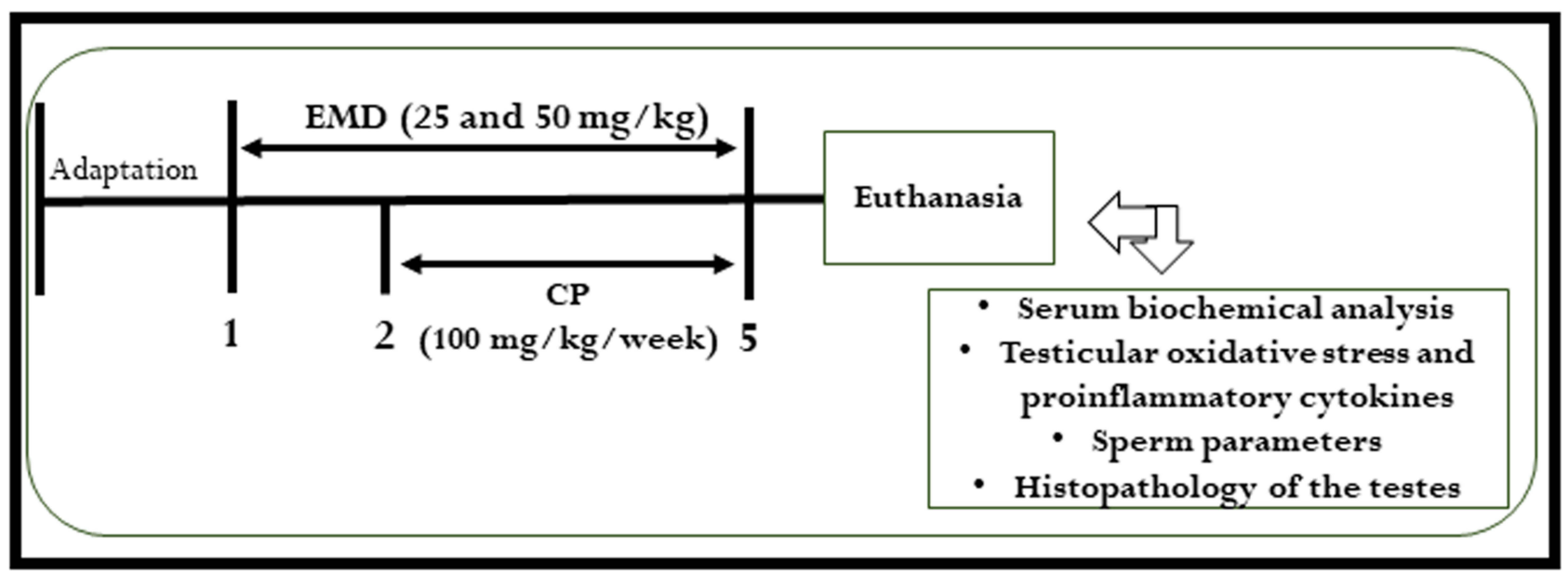

Figure I Study design diagram indicating the treatment protocol for evaluating the effect of emodin on cyclophosphamide-induced testicular toxicity.

EMD used in this study was based on earlier studies. ${ }^{24-26}$ At the end of the experiment, the rats were all anesthetised using thiopental sodium and blood were directly taken via cardiac puncture. The serum obtained from the whole blood after centrifugation was used for analysing reproductive hormones levels including testosterone, follicle-stimulating hormone (FSH) and luteinizing hormone (LH).

After euthanization, the testes and epididymis of the rats were dissected out, washed with distilled water and weighed. Thereafter, the testes were homogenized in phosphate buffered saline ( $\mathrm{pH} 7.4)$ at $6000 \mathrm{rpm}$ for $30 \mathrm{~min}$ at $4^{\circ} \mathrm{C}$, and the supernatant collected after centrifuging was preserved at $-80^{\circ} \mathrm{C}$ until further analysis.

\section{Histological Analysis}

The H\&E staining was performed on $10 \%$ buffered formalin fixed testes. The testes tissues were dehydrated in graded alcohol solutions and paraffinized using paraffin wax. The staining procedures followed standard H\&E staining protocol. ${ }^{27}$

\section{Evaluation of Sperm Count, Motility and Viability}

The estimation of epididymal sperm parameters including sperm count, motility and viability were in accordance with previous studies. ${ }^{27}$

\section{Assessment of Testicular Antioxidant Biomarkers}

The post-mitochondrial portion of the centrifuged testicular tissues was utilized for the estimation of antioxidant parameters and lipid peroxidation including catalase (CAT), glutathione peroxidase (GPx), glutathione (GSH), superoxide dismutase (SOD) and malonaldehyde (MDA) levels using kits procured from Nanjing Jiancheng Bioengineering Institute (Nanjing, China). The manufacturer's procedure included in the kit was strictly followed for the analysis.

\section{Assessment of Testicular Proinflammatory Mediators}

Proinflammatory cytokines including interlukin-6 (IL-6), and tumor necrosis factor-alpha $(\mathrm{TNF}-\propto)$ were analysed in the post-mitochondrial portion of the testes tissue homogenate with ELISA kits following the manufacturer's manual (Abcam, Cambridge, UK).

\section{Statistical Analysis}

Data were analysed using one way AVONA together with Newman-Keuls post hoc test with Graph Pad Prism (version 5). Results were shown as mean \pm SD. $p<0.05$ was considered as statistical significance.

\section{Results}

\section{Ameliorative Effect of EMD on Testes Weight}

The effect of treatment with EMD on the testes weight of $\mathrm{CP}$ treated rats is shown in Table 1. At the point of sacrifice, the testes weight of the CPCG rats was significantly ( $p<0.001)$ lower than the NCG. On the other hand, treatment with EMD significantly $(\mathrm{p}<0.001)$ increased the testes weights of the treated rats compared to CPCG. 
Table I Effect of EMD on the Markers of Testicular Function in CP Treated Rats

\begin{tabular}{|l|l|l|l|l|}
\hline Parameters/Groups & NCG & CPPG & EMD25+CP & EMD50+CP \\
\hline Testes weight (g) & $3.55 \pm 0.13$ & $2.30 \pm 0.12^{\dagger}$ & $3.50 \pm 0.07^{* *}$ & $3.36 \pm 0.29 * *$ \\
Epididymis weight (g) & $1.51 \pm 0.11$ & $1.01 \pm 0.09^{\dagger}$ & $1.31 \pm 0.11^{* *}$ & $1.37 \pm 0.11^{* *}$ \\
Sperm count $\left(\times 10^{6}\right)$ & $70.11 \pm 3.66$ & $38.03 \pm 6.34^{\dagger}$ & $57.05 \pm 5.60^{* *}$ & $60.33 \pm 3.08^{* *}$ \\
Sperm viability (\%) & $74.41 \pm 5.69$ & $37.40 \pm 4.52^{\dagger}$ & $55.36 \pm 5.15^{* *}$ & $62.36 \pm 4.79 * *$ \\
Sperm motility (\%) & $81.63 \pm 4.80$ & $40.11 \pm 7.72^{\dagger}$ & $63.06 \pm 5.46 * *$ & $66.80 \pm 5.67 * *$ \\
\hline
\end{tabular}

Notes: Values were indicated as mean \pm SD of 6 rats. ${ }^{\dagger} \mathrm{p}<0.001$ versus NCG; ${ }^{* *} \mathrm{p}<0.001$ versus CPCG; $* * * p<0.01$ versus CPCG.

Ameliorative Effects of EMD on Sperm Functional Parameters

The intraperitoneal administration of $\mathrm{CP}$ notably reduced sperm viability $(\mathrm{p}<0.001)$, motility $(\mathrm{p}<0.001)$ and sperm count $(\mathrm{p}<0.001)$ in the CPG group compared to the normal control rats (Table 1). Whereas, the sperm count, sperm motility and sperm viability were significantly improved in the EMD administered groups compared to CPCG group (Table 1).

\section{Ameliorative Effects of EMD on Serum Reproductive Hormones}

As depicted in Figure 2, the serum concentrations of hormones including testosterone, $\mathrm{LH}$ and $\mathrm{FSH}$ in the $\mathrm{CP}$-injected rats
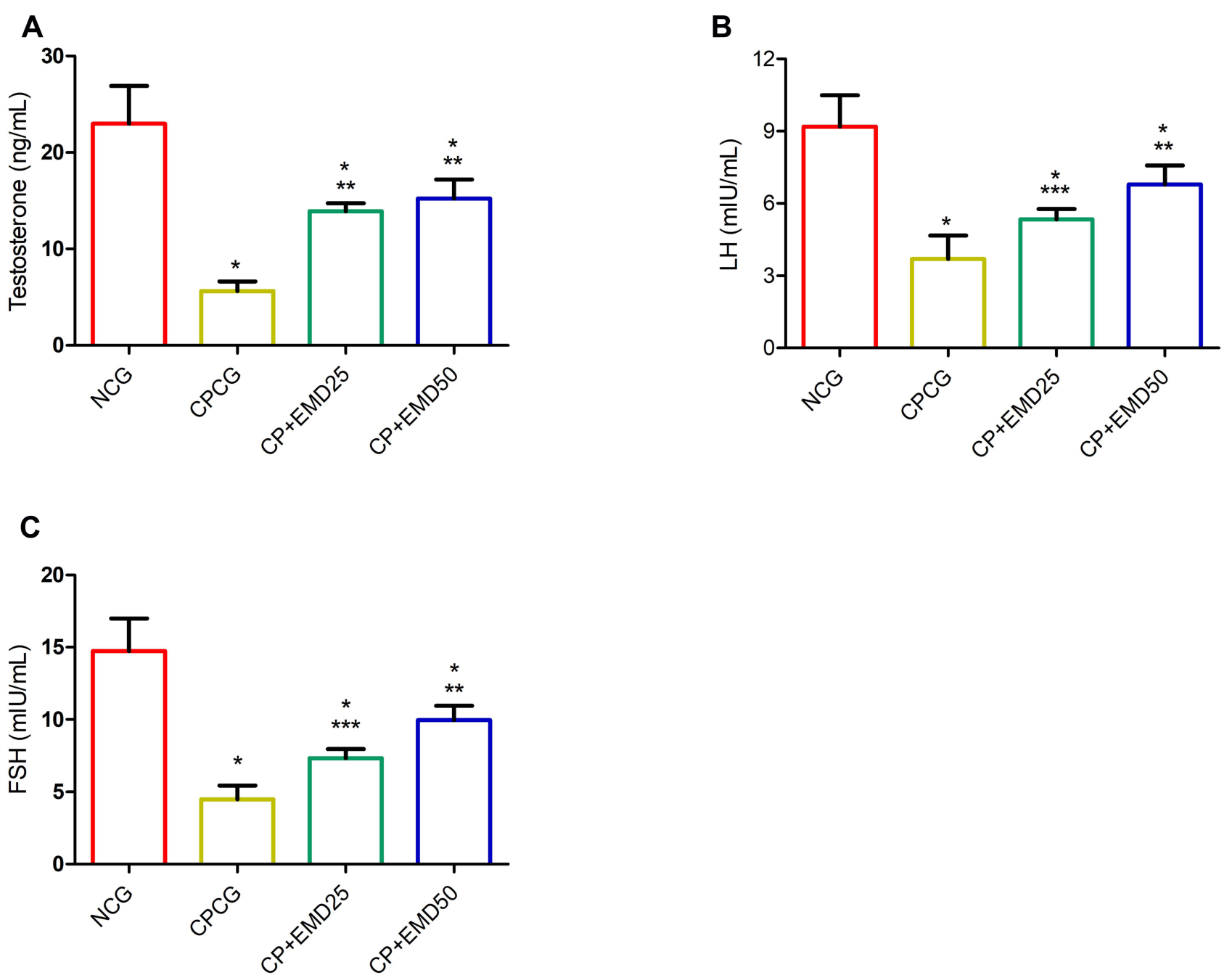

Figure 2 Effects of EMD on serum hormonal levels in CP treated rats. (A) testosterone (B) luteinizing hormone (LH)and (C) follicle-stimulating hormone (FSH) levels. Values were indicated as mean \pm SD of 6 rats. ${ }^{*} p<0.001$ versus NCG; ${ }^{*} p<0.001$ versus CPCG; $* * *<<0.01$ versus CPCG. 

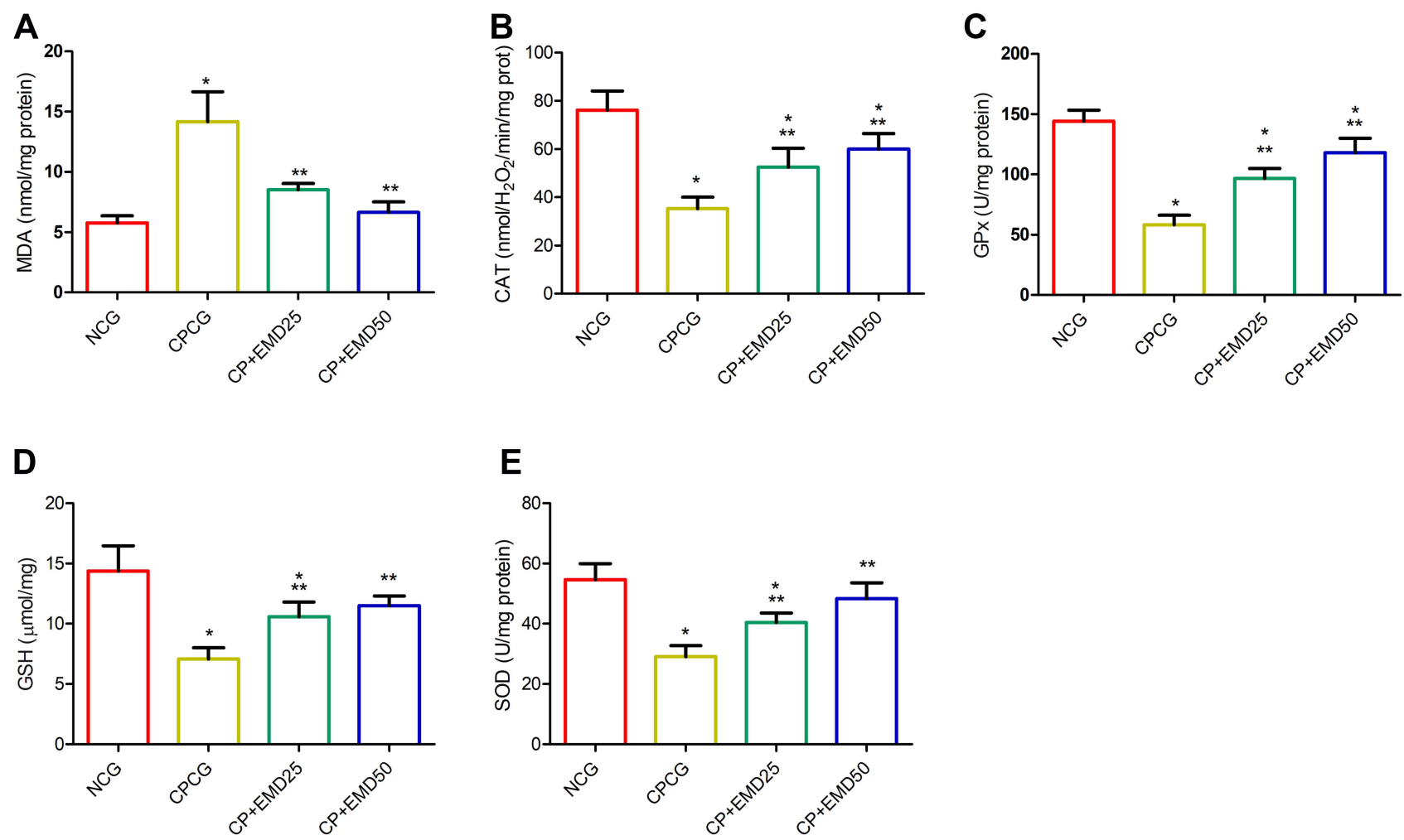

Figure 3 Effects of EMD on testicular oxidative stress levels in CP treated rats. (A) malonaldehyde (B) catalase (C) glutathione peroxidase (D) glutathione and (E) superoxide dismutase levels. Values were indicated as mean \pm SD of 6 rats. ${ }^{*} p<0.001$ versus NCG; $* * p<0.001$ versus CPCG; $* * * p<0.01$ versus CPCG.

were notably $(\mathrm{p}<0.001)$ reduced relative to the normal control. Conversely, in the EMD $+\mathrm{CP}$ groups, marked increases $(\mathrm{p}<$ $0.001)$ in the concentrations of testosterone, FSH and LH were observed relative to the untreated CPCG group (Figure 2A-C).

\section{Ameliorative Effects of EMD on Testicular Oxidative Stress Biomarkers}

Compared with the NCG, the testicular MDA concentration of the CPCG rats was significantly higher $(p<0.001)$. After intragastric treatment with EMD for 5 weeks, the MDA level of rats injected with CP was markedly decreased ( $p<$ 0.001) compared to the CPCG (Figure 3A). Meanwhile, the activities of CAT, GPx, GSH and SOD in the testes of the CPCG animals were notably decreased in response to $\mathrm{CP}$ administration (Figure 3B-E). In contrast, the intragastric administration of EMD markedly $(\mathrm{p}<0.001)$ impeded the decrease in SOD, GSH, GPx and CAT activities in comparison with CPCG group (Figure 3B-E).

\section{Ameliorative Effect of EMD on}

\section{Proinflammatory Cytokines in the Testes}

The testicular proinflammatory profile is shown in

Figure 4. The intra-testicular concentration of TNF- $\alpha$ and
IL-6 was significantly increased in the CPCG group relative to normal control group. Whereas, the administration of EMD significantly decreased testicular proinflammatory cytokine levels compared to CPCG.

\section{Effect of EMD on Histological Examination of the Testes}

In this study, the results of the histological assessment of the NCG testes indicated normal seminiferous tubule, connective tissue, germinal epithelial cells, interstitial cells of Leydig and obvious spermatids. The representative testicular tissues histology of the CPCG showed significant anomalies including loss of seminiferous tubule, connective tissues as well as damages to the germinal epithelium and spermatocytes. Whereas, in the EMD + CP groups, marked changes were observed in the histopathological alterations induced by $\mathrm{CP}$ following treatment with EMD (Figure 5).

\section{Discussion}

Gonadotoxicity is one of the most prominent and most frequently encountered side effect associated with prolonged use of $\mathrm{CP}$ and there is an urgent need to discover therapeutic means to mitigate or preserve the reproductive 
A

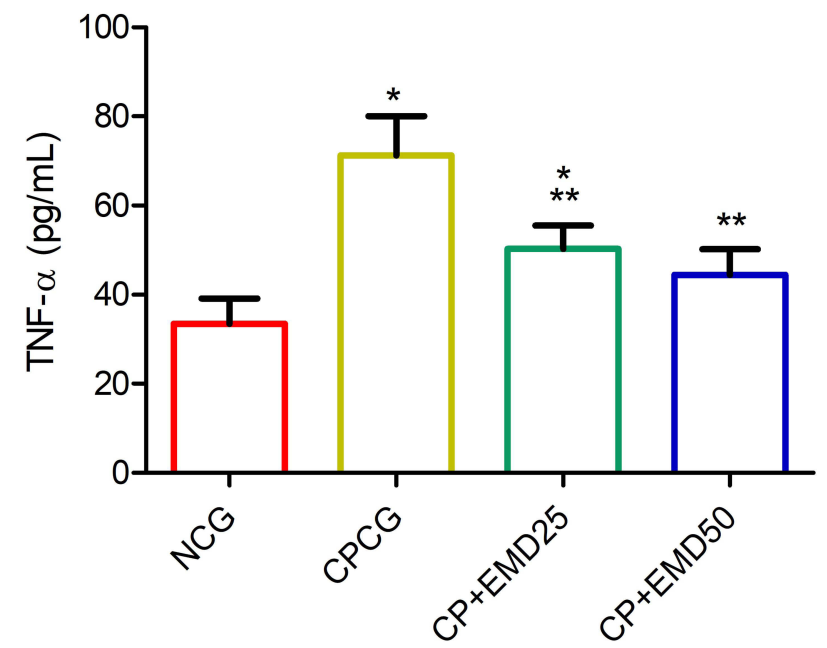

B

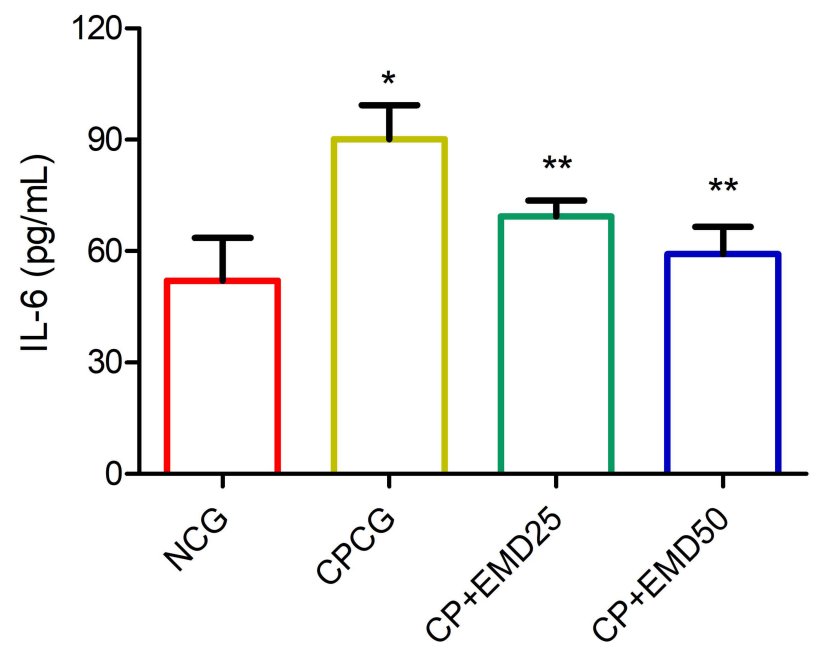

Figure 4 Effects of EMD on testicular proinflammatory cytokine levels in CP treated rats. (A) tumor necrosis factor alpha and (B) interleukin 6. Values were indicated as mean \pm SD of 6 rats. ${ }^{*} \mathrm{p}<0.001$ versus NCG; $* * p<0.001$ versus CPCG; $* * * p<0.01$ versus CPCG.

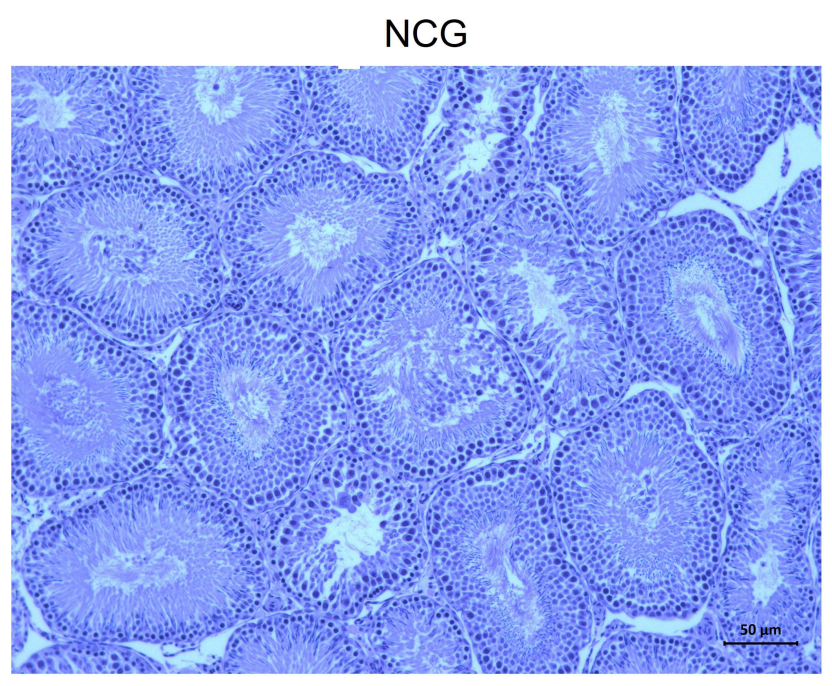

\section{$\mathrm{CP}+\mathrm{EMD} 25$}

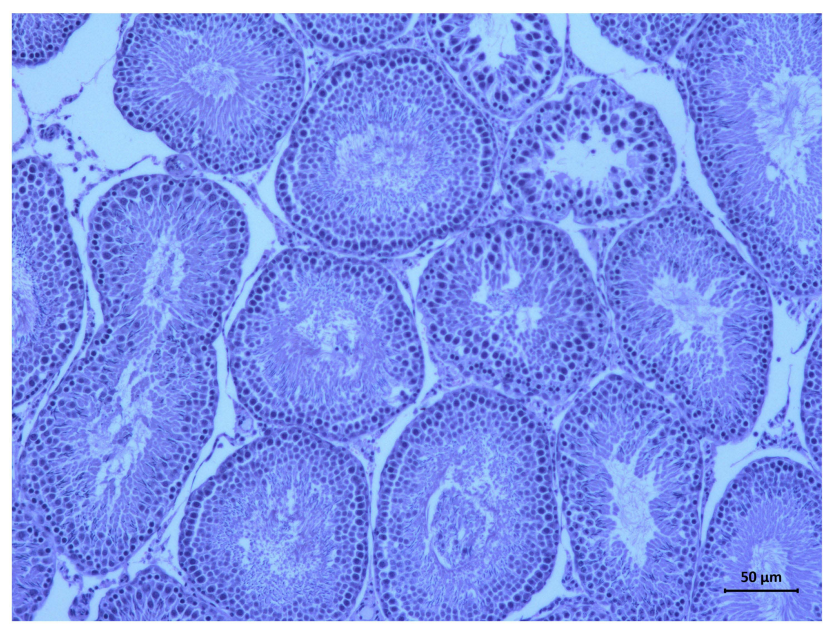

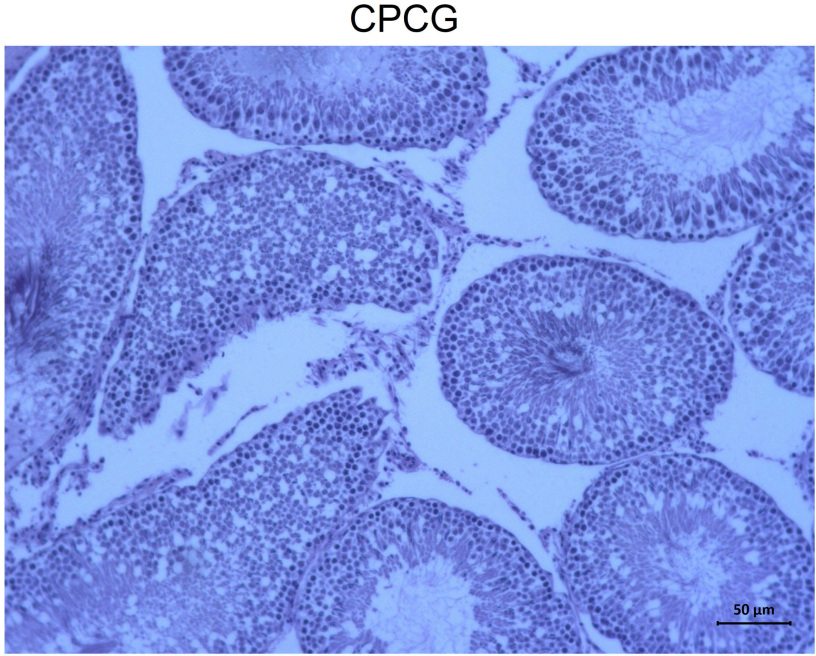

CP+EMD50

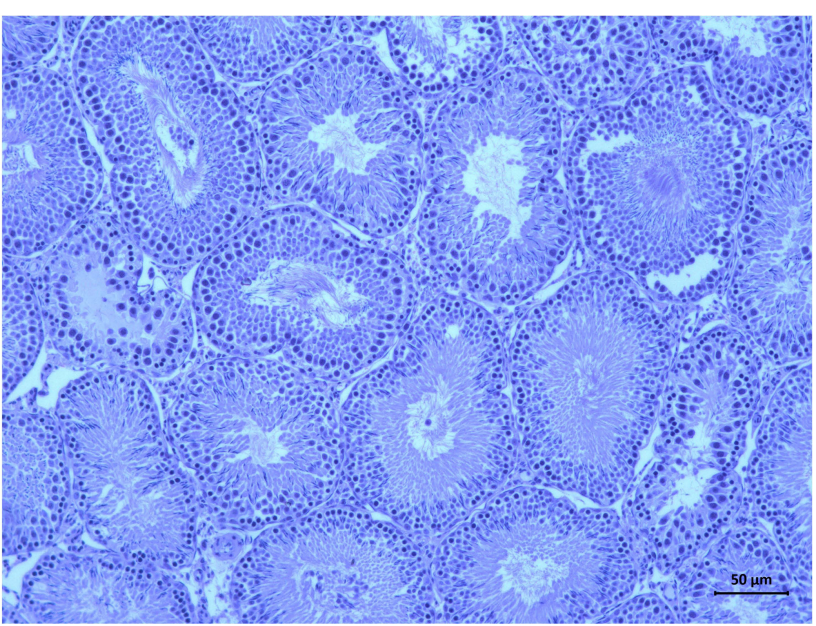

Figure 5 Representative histopathological sections of the testes showing the effect of EMD on CP induced histological alterations. H\&E staining; $20 \times$ magnification; Scale bar $=50 \mu \mathrm{m}$. Black arrow: loss of connective tissues; Red arrow: loss of seminiferous tubule; Brown arrow: damages to the germinal epithelium and spermatocytes. 
viability of cancer patients exposed to $\mathrm{CP}$ treatment. This study was aimed at unravelling the gonado-protective effects of emodin against CP-induced gonadotoxicity in male rats by assessing the impact of the treatment on redox, inflammatory, histological and endocrine alterations. The results indicated that the intraperitoneal administration of $\mathrm{CP}$ engender significant testicular oxidative stress, inflammation and histological damages. Moreover, treatment with emodin markedly reversed these aforementioned changes in the testes.

The toxicity of CP has been widely linked to the toxic metabolites that are generated upon enzymatic conversion of the drug by cytochrome P450 (CYP) enzymes. ${ }^{28,29}$ Acrolein, a reactive electrophilic molecule produced from $\mathrm{CP}$ metabolism has been principally implicated in most of the oxidative damage associated with CP administration. Acrolein has the ability to instigate ROS and oxidative damages via DNA cross link and interference with tissues that rapidly proliferate. ${ }^{2,30}$ In addition to the accrued ROS and oxidative stress generated upon CP exposure, several studies have illustrated that $\mathrm{CP}$ also have the ability to depress antioxidant enzymes ability. ${ }^{12,31,32}$ The rapidly proliferating ability of testicular tissue makes them vulnerable to the toxicity of $\mathrm{CP}^{33}$ In corroboration with earlier studies, our study portrayed that the intraperitoneal injection of CP led to drastic increase in oxidative stress indices in the testes as demonstrated by high MDA level and corresponding depression in testicular antioxidant enzyme capabilities (SOD, GSH, CAT and GPx). ${ }^{34-36}$ The administration of EMD markedly attenuated testicular MDA level, while simultaneously increasing testicular GSH, SOD, CAT and GPx activities compared with the CP group. This effect may be obviously related to the antioxidant efficacy of EMD which is supported by previous studies. $^{22}$

The male reproductive system is comprised of several hormones that enhances the effective production of testosterone. Specifically, LH and FSH are critically involved in the production of testosterone through the stimulation of Leydig cells and spermatogenesis. ${ }^{36}$ Furthermore, studies have demonstrated that the integrity of sperm parameters including sperm count, viability and motility are directly related to the rectitude of steroidogenic and spermatogenic pathways. ${ }^{36}$ Meanwhile, ROS and oxidative stress have been implicated in the reduction of steroidogenic hormones including 17- $\beta$ hydroxysteroid dehydrogenase and 3- $\beta$-hydroxysteroid dehydrogenase enzymes, leading to depression of testosterone and LH production. ${ }^{37-39}$ In addition, the reduction in the weight of the testes of $\mathrm{CP}$ treated rats suggests a decrease in the quantity of spermatogenic cells, which has been suggested to indirectly impair the secretion of testosterone, gonadotropins and spermatogenesis. ${ }^{12}$ The results from this study indicated significant reduction in serum concentrations of testosterone, FSH and LH of CP treated animals, while treatment with EMD significantly increased the concentration of FSH, LH and testosterone in the treated groups. This results were clearly supported by the histopathological examination, which revealed gross decline and necrosis of cells involved in production of testosterone and spermatogenesis. ${ }^{31,36}$

ROS and oxidative stress mediated inflammation has been implicated in the pathophysiology of CP-induced toxicity including testicular damages. ${ }^{29,34}$ The increased testicular levels of TNF- $\alpha$ and IL- 6 in the untreated CP administered rats suggests the instigation of inflammatory related pathways, which corroborates well with previous reports. ${ }^{40}$ Antioxidant and anti-inflammatory agents have been illustrated for their protective capabilities against chemotherapy induced testicular toxicities partly or wholly due to their radicals scavenging capabilities and their modulatory roles on oxidative sensitive inflammatory pathways. ${ }^{5,41}$ The decrease in testicular TNF- $\alpha$ and IL-6 levels following the intragastric administration of EMD suggests an improvement in the testicular proinflammatory mediator status in $\mathrm{CP}$ treated rats which corroborates previous reports. $5,29,34$

The undesirable consequences of $\mathrm{CP}$ on the reproductive system have been extensively highlighted in many reports. ${ }^{12,13,37}$ The underlying factor associated with CP toxicity is mainly its ability to generate ROS and oxidative stress which subsequently impairs endocrine and gonadotropin secretion resulting in reduction of sex hormones, altered semen quality and decreased male fertility. In addition, CP-induced ROS perturbs spermatogenesis via the testicular-endocrine axis which alters the production of follicle stimulating hormone, luteinizing hormone causing subnormal levels of testosterone. ${ }^{32}$ As such, based on the seemingly extensive and prominent status of ROS and oxidative stress status in CP toxicity and the resulting decline in steroidogenesis, testosterone production, sperm functional parameters and testicular antioxidant mechanism, the therapeutic effects of EMD may thus be hinged on its ability to modulate oxidative imbalance, counteract ROS and oxidative stress, which subsequently results in increase in endocrine function and spermatogenesis culminating in improved reproductive function. 


\section{Conclusion}

Conclusively, this is the first study that demonstrated that the intragastric administration of emodin at doses of 25 and $50 \mathrm{mg} / \mathrm{kg}$ prevented oxidative damage induced by $\mathrm{CP}$ and alleviated testicular and sperm parameters following CP injection. The protective effect was mediated by restoration of testicular antioxidant prowess, serum endocrine concentration (testosterone, FSH and LH), amelioration of histopathological alterations and testicular inflammation in CP-treated rats. As such, emodin may serve as a dietary therapeutic supplement to protect against chemotherapy induced testicular and reproductive toxicity.

\section{Disclosure}

The authors declare no conflicts of interest for this work.

\section{References}

1. Lehmann F, Wennerberg J. Evolution of nitrogen-based alkylating anticancer agents. Processes. 2021;9:377. doi:10.3390/pr9020377

2. Sung H, Ferlay J, Siegel RL, et al. Global Cancer Statistics 2020: GLOBOCAN estimates of incidence and mortality worldwide for 36 cancers in 185 countries. CA Cancer J Clin. 2021;71:209-249. doi: $10.3322 /$ caac. 21660

3. Falzone L, Salomone S, Libra M. Evolution of cancer pharmacological treatments at the turn of the third millennium. Front Pharmacol. 2018;9:1300.

4. Zhou J, Kang Y, Chen L, et al. The drug-resistance mechanisms of five platinum-based antitumor agents. Front Pharmacol. 2020;11:343. doi:10.3389/fphar.2020.00343

5. Wang L, He Y, Li Y, et al. Protective effects of nucleosides-rich extract from Cordyceps cicadae against cisplatin induced testicular damage. Chem Biodivers. 2020;17:e2000671.

6. Nurgali K, Jagoe RT, Abalo R. Editorial: adverse effects of cancer chemotherapy: anything new to improve tolerance and reduce sequelae? Front Pharmacol. 2018;9:245. doi:10.3389/fphar.2018.00245

7. Mittra I, Pal K, Pancholi N, et al. Prevention of chemotherapy toxicity by agents that neutralize or degrade cell-free chromatin. Ann Oncol. 2017;28:2119-2127. doi:10.1093/annonc/mdx318

8. Akomolafe SF, Aluko BT. Protective effect of curcumin on fertility in cyclophosphamide exposed rats: involvement of multiple pathways. J Food Biochem. 2020;44:e13095. doi:10.1111/jfbc.13095

9. Madondo MT, Quinn M, Plebanski M. Low dose cyclophosphamide: mechanisms of T cell modulation. Cancer Treat Rev. 2016;42:3-9. doi:10.1016/j.ctrv.2015.11.005

10. Spears N, Lopes F, Stefansdottir A, et al. Ovarian damage from chemotherapy and current approaches to its protection. Hum Reprod Update. 2019;256:673-693. doi:10.1093/humupd/dmz027

11. Huang YP, Liu W, Liu YD, et al. Right testicular volume is a dominant predictor of testicular function determined by sperm parameters and total testosterone. Andrologia. 2018;50:e12955. doi:10.1111/and.12955

12. Ghobadi E, Moloudizargari M, Asghari MH, Abdollahi M. The mechanisms of cyclophosphamide-induced testicular toxicity and the protective agents. Expert Opin Drug Metab Toxicol. 2017;13:525-536. doi:10.1080/17425255.2017.1277205

13. Iqubal A, Syed MA, Najmi AK, Ali J, Haque SE. Ameliorative effect of nerolidol on cyclophosphamide-induced gonadal toxicity in Swiss Albino mice: biochemical-, histological- and immunohistochemical-based evidences. Andrologia. 2020;52:e13535. doi:10.1111/and.13535
14. Zhang QY, Wang FX, Jia KK, Kong LD. Natural product interventions for chemotherapy and radiotherapy-induced side effects. Front Pharmacol. 2018;9:1253. doi:10.3389/fphar.2018.01253

15. Stankovic JSK, Selakovic D, Mihailovic V, Rosic G. Antioxidant supplementation in the treatment of neurotoxicity induced by platinum-based chemotherapeutics - a review. Int $J$ Mol Sci. 2020;21:7753. doi:10.3390/ijms21207753

16. Liu YQ, Wang XL, He DH, Cheng YX. Protection against chemotherapy- and radiotherapy-induced side effects: a review based on the mechanisms and therapeutic opportunities of phytochemicals. Phytomedicine. 2021;80:153402.

17. Fusco R, Salinaro AT, Siracusa R, et al. Hidrox ${ }^{\circledR}$ counteracts cyclophosphamide-induced male infertility through NRF2 pathways in a mouse model. Antioxidants. 2021;10:778. doi:10.3390/antiox10050778

18. Semwal RB, Semwal DK, Combrinck S, Viljoen A. Emodin a natural anthraquinone derivative with diverse pharmacological activities. Phytochemistry. 2021;190:112854.

19. Dang S, Zhang Z, Yuan L, et al. Effects of emodin and astragalus polysaccharides on the expressions of GST-P and TGF- $\beta 1$ in rats' hepatocellular carcinoma constitution. $J$ Xi'an Jiaotong Univ Med Sci. 2006;27:250-253.

20. Dang SS, Zhang X, Jia XL, et al. Protective effects of emodin and astragalus polysaccharides on chronic hepatic injury in rats. Chin Med J. 2008;121:1010-1014. doi:10.1097/00029330-200806010-00009

21. Ali BH, Al-Salam S, Al Husseini IS, et al. Abrogation of cisplatin-induced nephrotoxicity by emodin in rats. Fundam Clin Pharmacol. 2013;27:192-200. doi:10.1111/j.1472-8206.2011.01003.x

22. Zhao Z, Liu B, Ge X, et al. Emodin attenuates CY-induced oxidative injury in PBLs of the blunt snout bream (Megalobrama amblycephala) though the Nrf2-Keap1 signaling pathway. Aquaculture. 2021;545:737201. doi:10.1016/j.aquaculture.2021.737201

23. Liu C, Ma M, Wen C, Uz Zaman R, Olatunji OJ. Antiallodynic and anti-hyperalgesia effects of Tiliacora triandra against cisplatin-induced peripheral neuropathy. All Life. 2021;14:441-449. doi:10.1080/26895293.2021.1927204

24. Wang H, Olatunji OJ, Xue N. Antinociceptive, anti-hyperalgesia and antiallodynic activities of polyphenol rich extract from Shorea roxburghii against cyclophosphamide induced peripheral neuropathy. Chem Biodivers. 2021;18:e2100415.

25. Shen C, Pan Z, Wu S, et al. Emodin palliates high-fat diet-induced nonalcoholic fatty liver disease in mice via activating the farnesoid X receptor pathway. $J$ Ethnopharmacol. 2021;279:114340. doi:10.1016/j.jep.2021.114340

26. Gao Z, Sui J, Fan R, Qu W, Dong X, Sun D. Emodin protects against acute pancreatitis-associated lung injury by inhibiting NLPR3 inflammasome activation via Nrf2/HO-1 signaling. Drug Des Devel Ther. 2020;14:1971-1982. doi:10.2147/DDDT.S247103

27. Zhao LL, Makinde EA, Olatunji OJ. Protective effects of ethyl acetate extract from Shorea roxburghii against diabetes induced testicular damage in rats. Environ Toxicol. 2021;36:374-385. doi:10.1002/tox.23043

28. Jeelani R, Khan SN, Shaeib F, et al. Cyclophosphamide and acrolein induced oxidative stress leading to deterioration of metaphase II mouse oocyte quality. Free Radic Biol Med. 2017;110:11-18. doi:10.1016/j.freeradbiomed.2017.05.006

29. Iqubal A, Sharma S, Ansari MA, et al. Nerolidol attenuates cyclophosphamide-induced cardiac inflammation, apoptosis and fibrosis in Swiss Albino mice. Eur J Pharmacol. 2019;863:172666. doi:10.1016/j.ejphar.2019.172666

30. Mahipal P, Pawar RS. Nephroprotective effect of Murraya koenigii on cyclophosphamide induced nephrotoxicity in rats. Asian Pac J Trop Med. 2017;10:869-873. doi:10.1016/j.apjtm.2017.08.005

31. Ekeleme-Egedigwe CA, Famurewa AC, David EE, Eleazu CO, Egedigwe UO. Antioxidant potential of garlic oil supplementation prevents cyclophosphamide-induced oxidative testicular damage and endocrine depletion in rats. $J$ Nutr Intermed Metab. 2019;18:100109. doi:10.1016/j.jnim.2020.100109 
32. Nayak G, Rao A, Mullick P, et al. Ethanolic extract of Moringa oleifera leaves alleviate cyclophosphamide-induced testicular toxicity by improving endocrine function and modulating cell specific gene expression in mouse testis. J Ethnopharmacol. 2020;259:112922. doi:10.1016/j.jep.2020.112922

33. Aguilar-Mahecha A, Hales BF, Robaire B. Acute cyclophosphamide exposure has germ cell specific effects on the expression of stress response genes during rat spermatogenesis. Mol Reprod Dev. 2001;60:302-311. doi:10.1002/mrd.1092

34. Rezaei S, Hosseinimehr SJ, Zargari M, Karimpour Malekshah A, Mirzaei M, Talebpour Amiri F. Protective effects of sinapic acid against cyclophosphamide-induced testicular toxicity via inhibiting oxidative stress, caspase-3 and NF-kB activity in BALB/c mice. Andrologia. 2021;53(10). doi:10.1111/and.14196

35. Cengiz M, Sahinturk V, Yildiz SC, et al. Cyclophosphamide induced oxidative stress, lipid per oxidation, apoptosis and histopathological changes in rats: protective role of boron. J Trace Elem Med Biol. 2020;62:126574. doi:10.1016/j.jtemb.2020.126574

36. Oyagbemi AA, Omobowale TO, Saba AB, et al. Gallic acid protects against cyclophosphamide-induced toxicity in testis and epididymis of rats. Andrologia. 2016;48:393-401. doi:10.1111/and.12459

37. Potnuri AG, Allakonda L, Lahkar M. Crocin attenuates cyclophosphamide induced testicular toxicity by preserving glutathione redox system. Biomed Pharmacother. 2018;101:174-180. doi:10.1016/j. biopha.2018.02.068
38. Ghosh D, Das UB, Misro M. Protective role of $\alpha$-tocopherolsuccinate (provitamin-E) in cyclophosphamide induced testicular gametogenic and steroidogenic disorders: a correlative approach to oxidative stress. Free Radic Res. 2002;36:1209-1218. doi:10.1080/ 1071576021000016472

39. Ghosh D, Das UB, Ghosh S, Mallick M, Debnath J. Testicular gametogenic and steroidogenic activities in cyclophosphamide treated rat: a correlative study with testicular oxidative stress. Drug Chem Toxicol. 2002;25:281-292. doi:10.1081/DCT-120005891

40. Ujah GA, Nna VU, Suleiman JB, et al. Tert-butylhydroquinone attenuates doxorubicin-induced dysregulation of testicular cytoprotective and steroidogenic genes, and improves spermatogenesis in rats. Sci Rep. 2021;11:5522. doi:10.1038/s41598-021-85026-7

41. Temel Y, Kucukler S, Yıldırım S, Caglayan C, Kandemir FM. Protective effect of chrysin on cyclophosphamide-induced hepatotoxicity and nephrotoxicity via the inhibition of oxidative stress, inflammation, and apoptosis. Naunyn Schmiedebergs Arch Pharmacol. 2020;393:325-337. doi:10.1007/s00210-019-01741-z
Drug Design, Development and Therapy

\section{Publish your work in this journal}

Drug Design, Development and Therapy is an international, peerreviewed open-access journal that spans the spectrum of drug design and development through to clinical applications. Clinical outcomes, patient safety, and programs for the development and effective, safe, and sustained use of medicines are a feature of the journal, which has also

\section{Dovepress}

been accepted for indexing on PubMed Central. The manuscript management system is completely online and includes a very quick and fair peer-review system, which is all easy to use. Visit http://www. dovepress.com/testimonials.php to read real quotes from published authors. 